\title{
Clinical Observation of Laparoscopic Anatomic Hepatectomy for Early Primary Liver Cancer
}

\author{
Yan Shaoxiong ${ }^{1 \mathrm{a}}$, Shen Shiheng ${ }^{1, b, *}$ \\ ${ }^{1}$ Zhaotong Health Vocational Colleage 657000
}

\begin{abstract}
At present, liver resection is the most effective treatment for malignant liver tumors, and with the rapid development of medical technology, anatomical liver resection has been widely used in clinical practice. This paper mainly studies the clinical effect of laparoscopic anatomic hepatectomy in the treatment of early primary liver cancer. According to the surgical method, the patients were divided into anatomic and nonanatomic hepatectomy groups. Preoperative, intraoperative and postoperative efficacy data of the two groups were analyzed. According to the clinical results, the anatomical resection of liver cancer has short operation time, fewer postoperative complications, fast recovery of liver function, and long survival time without tumor, with obvious clinical effect and definite curative effect, which is worthy of promotion.
\end{abstract}

\section{Introduction}

Primary liver cancer is the most common liver malignant tumor in China. In patients with cirrhosis, nearly $80-90 \%$ of patients will further develop HCC. It is currently believed that the pathogenesis of hepatocellular carcinoma is closely related to chronic viral infections, including hepatitis $\mathrm{B}$ virus (HBV) and hepatitis $\mathrm{C}$ virus (HCV) [1]. In addition, cirrhosis caused by chronic alcoholism is also closely related. The number of hepatitis patients in China ranks the first in the world. Every year, a large number of hepatitis patients develop into liver cancer on the basis of cirrhosis. According to statistics, the number of new cases of liver cancer in China accounts for more than $50 \%$ of the global total. Due to the accumulation of surgical experience of hepatobiliary surgeons and the progress of surgical instruments, the operation mode of hepatobiliary surgery has changed greatly in the past twenty years. With the extensive promotion of laparoscopic technology, more and more traditional open surgery has been replaced by laparoscopic surgery, and laparoscopic surgery has become the mainstream way of liver surgery in some hospitals.

In recent years, with the continuous understanding and deepening of the anatomical knowledge of the liver by surgeons, the continuous improvement of surgical skills and the continuous refinement of operating instruments, anatomical hepatectomy may become the first choice. In A retrospective study, Kon et al. showed that in patients with child-pugh grade A liver function, intraoperative anatomical hepatectomy was more prudent, postoperative liver function recovery was faster and the incidence of liver failure was lower, but extended hepatectomy was contraindicated for patients with significant liver function impairment [2]. Nanashima found that in the patients who underwent anatomic and non-anatomic hepatectomy, tumor diameter was the main factor affecting the postoperative tumor-free survival rate and tumor-free survival time of the two groups of patients. According to the analysis, anatomic hepatectomy showed significant advantages when the tumor diameter was between $2 \mathrm{~cm}$ and $5 \mathrm{~cm} \mathrm{[3].}$

In this paper, clinical data of patients with single liver cancer treated by surgery in our hospital were collected and divided into anatomic hepatectomy group and nonanatomic hepatectomy group according to different surgical methods. Preoperative treatment of patients in both groups; Intraoperative effect; Postoperative outcome data were collected to evaluate the advantages and disadvantages of the two surgical methods.

\section{Anatomical Hepatectomy for Early Primary Liver Cancer}

\subsection{Development of Laparoscopic Hepatectomy}

In recent years, endoscopic surgery has been widely recognized and applied not only in the field of abdominal surgery, but also in cardiovascular surgery, thoracic surgery, breast surgery, pediatric surgery, gynecology, urology and orthopedics. Endoscopic surgery performs better in some suitable procedures because of its greater field of vision, less invasive and cosmetic effect on the incision. At present, some endoscopic surgery has become the first-line treatment for some diseases. Laparoscopic cholecystectomy has become the gold standard in abdominal surgery. Since the early 1990s, laparoscopic cholecystectomy has gained rapid application worldwide. Since then, laparoscopic surgery has been gradually 
introduced to the liver cyst topodrainage, splenectomy, adrenal resection, hernia repair, anti-reflux surgery and bariatric surgery. Laparoscopic hepatectomy has been carried out in various medical centers in succession, but its application and worldwide progress has been relatively slow, mainly due to the difficulty in controlling bleeding during the separation of liver parenchyma, especially when most of the liver is removed, and the concern about positive surgical margins and tumor spread [4]. In recent years, a growing number of papers have been published on laparoscopic hepatectomy, and the use of this procedure has increased rapidly. Many previously published articles have demonstrated the safety and feasibility of laparoscopic hepatectomy.

At present, almost all hepatectomy is performed laparoscopically. However, most laparoscopic hepatectomies are performed by either non-anatomic or local resection. Laparoscopic local hepatectomy was reported to be the most common $(45 \%)$ in a review of 2,804 cases of laparoscopic hepatectomy performed in specialized medical centers worldwide. Laparoscopic hepatectomy is a better option than open surgery and can be seen as the "gold standard" approach. On the other hand, laparoscopic hepatectomy for most cases is progressing slowly worldwide and can only be performed in specialized centers by hepatobiliary surgeons with a wealth of minimally invasive techniques. However, in the past few years, with the improvement of laparoscopic technology, the development of new specialized technology and the emergence of hand-assisted laparoscopic surgery and other technologies, the limitations of most laparoscopic hepatectomy are being overcome [5].

Earlier, minimally invasive surgery was initially banned for use in malignant tumors because of concerns about the risk of inadequate tumor resection. In recent years, however, the risk of negative margins, recurrence of puncture holes, and tumor spread has been reassessed. As a result, it has been reported that laparoscopic surgery is no longer prohibited for malignant tumors.

Studies have shown that the incidence of postoperative complications such as surgically associated infection and refractory ascites is lower than that of open hepatectomy, the necessity of postoperative complications is also reduced, and the length of hospital stay is significantly shortened.

\subsection{Advantages and Disadvantages of Laparoscopic Hepatectomy}

Laparoscopic hepatectomy has some advantages over open hepatectomy:

Laparoscopic incisions are significantly smaller than open incisions, and the incisions are scattered, with little impact on abdominal wall tissue and damage, low incidence of incision fat liquefaction, incision infection and other complications, and quick wound healing.

Because the laparoscopic imaging system can enlarge the surgical field of vision and facilitate fine operation, it can effectively reduce intraoperative bleeding.
Laparoscopic hepatectomy is usually performed anatomic resection, which can significantly reduce the incidence of postoperative complications such as hemorrhage, biliary fistula, ascites, liver failure, and slow recovery of gastrointestinal function, etc., with less intraoperative stimulation to liver and gastrointestinal organs [6].

Less adhesion after laparoscopic surgery provides better conditions for further treatment.

The disadvantages of laparoscopic hepatectomy compared with open hepatectomy:

Compared with open hepatectomy, laparoscopic hepatectomy is more difficult to expose, more difficult to operate, and the level of surgeons is high.

In laparoscopic surgery, $\mathrm{CO} 2$ pneumoperitoneum should be established, which may cause gas embolism [7].

\subsection{Anatomical Hepatectomy}

\subsubsection{Advantages of Anatomical Hepatectomy}

Since HCC is mainly transmitted through vascular infiltration, anatomic hepatectomy based on the anatomical characteristics of the liver segment can theoretically complete the removal of tumor-related areas, including possible venous tumor thrombus around the tumor. The theoretical advantages of anatomic hepatectomy for HCC include improvement of intrahepatic metastasis by removal of portal venous thromboembolism, preservation of functional liver parenchyma, and a larger surgical margin. In addition, according to the boundary formed after regional occlusion, the liver parenchyma is broken off, and there is no large blood vessel or bile duct between the liver segments, so the amount of blood loss is significantly reduced [8-9]. However, it may increase the risk of postoperative liver insufficiency, especially in patients with cirrhosis. This is the current point of contention between AR and NAR.

\subsubsection{Application of Anatomical Hepatectomy}

In patients with liver cirrhosis, the liver artery blood supply was increased in compensation, and the bleeding probability was very high during surgical resection. Uncontrolled bleeding from the liver wound was often the main reason for the conversion of laparoscopic liver resection to the abdomen when non-anatomical major hepatectomy was performed. Laparoscopic anatomic hepatectomy emphasizes that the intraoperative blood flow to the liver should be cut off at the tumor site first, and then the partial liver, liver lobe or liver segment to be removed according to the anatomical relationship of the liver, and finally the liver tissue should be removed [10]. 


\section{Clinical Trial of Anatomic Hepatectomy}

\subsection{Subject Investigated}

In this study, 22 cases of laparoscopic hepatectomy and 20 cases of open resection were included in the laparoscopic group and the total open group, respectively.

\subsection{Inclusion and Exclusion Criteria}

\subsubsection{Inclusion Criteria}

According to the general condition of the patients and the operation, we developed the inclusion criteria for patients in the fluorescence group as follows: preoperative assessment of liver cancer stage, and inclusion of patients in stage A of Barcelona stage; Preoperative examination showed no distant metastasis; Preoperative assessment of child-pugh grade of liver function of patients, including patients of grade A or B; The patient had no previous history of abdominal surgery and no serious intraperitoneal adhesions. Maximum tumor diameter $<15 \mathrm{~cm}$; Signed informed consent and agreed to cooperate with follow-up.

\subsubsection{Exclusion Criteria}

Severe intraperitoneal adhesions, or tumors adjacent to or invading the diaphragm, inferior vena cava and other large vessels, resulting in the failure of successful separation under endoscopy; Preoperative examination has confirmed the presence of extrahepatic invasion or distant metastasis, or the presence of portal venous thrombus; Has been identified as secondary liver cancer patients; Patients with serious cardiovascular and cerebrovascular diseases, endocrine diseases such as severe diabetes, and diseases of the immune system; The coagulation function was obviously abnormal; Preoperative evaluation indicated that postoperative residual liver volume was insufficient, postoperative liver failure was high risk, and hemihepatectomy was not suitable. Abnormal metabolism or excretion of bile.

\subsection{Surgical Method}

Trocar was implanted with an operating instrument to detect the presence of ascites in the abdominal cavity, metastatic nodules in various organs outside the liver and the greater omentum, as well as the size, color, texture of the liver and the adhesion of the lesion to the surrounding area. Fully free the perihepatic duct, fully exposed lesions, intraoperative using ultrasonic knife from the liver before facing backward, from the bottom up, from shallow to deep from the liver parenchyma, adopting Hemolock clip after cut off, according to the intraoperative situation, necessary: liver door block, liver tumor resection or combining cutting closer anatomical sex liver resection, liver wound to the bipolar electric coagulation or electric knife bleeding, after fully hemostatic and flushing, placed hemostatic gauze, drainage tube is placed in the corresponding operation position, will from the poke holes on the right side of the drainage tube in vitro. According to the size of the resected specimen, select the appropriate disposable specimen bag, enlarge one of the puncture hole incision, and then take out the specimen.

\subsection{Test}

The T-test formula used in this paper is as follows:

$$
\begin{aligned}
& t=\frac{\overline{X-\mu}}{\frac{\sigma_{X}}{\sqrt{n-1}}} \\
& t=\frac{\overline{X-\mu}}{\frac{\sigma_{X}}{\sqrt{n}}}
\end{aligned}
$$

\section{Clinical Results of anatomic Hepatectomy}

\subsection{Comparison of Surgical Outcomes between the Two Groups of HCC Patients}

Table1. Surgical conditions of HCC patients in the two groups

\begin{tabular}{|c|c|c|}
\hline Observation target & AR group & NAR group \\
\hline Incisal edge $(\mathrm{cm})$ & 1.4 & 1.5 \\
\hline $\begin{array}{c}\text { Time of } \\
\text { operation }(\mathrm{min})\end{array}$ & 179.86 & 132.36 \\
\hline $\begin{array}{c}\text { Amount of } \\
\text { bleeding }(\mathrm{ml})\end{array}$ & 337.33 & 424.89 \\
\hline Hospital stays(d) & 22.21 & 20.53 \\
\hline
\end{tabular}

As shown in Table 1, the surgical margin distance of the anatomical hepatectomy group was $1.4 \mathrm{~cm}$, and the length of hospital stay was $22.21 \mathrm{~d}$, while that of the nonanatomical group was $1.5 \mathrm{~cm}$ and the length of hospital stay was $20.53 \mathrm{~d}$. After $\mathrm{t}$ test, the difference between the two groups was not statistically significant $(\mathrm{P}>0.05)$. The operative time of the anatomic liver resection group was $179.86 \mathrm{~min}$, which was significantly higher than that of the non-anatomic liver resection group (132.36min, $\mathrm{P}<0.05)$, and the difference between the two groups was statistically significant $(\mathrm{P}<0.05)$. The intraoperative blood loss in the two groups was $33.733 \mathrm{ml}$ and $424.89 \mathrm{ml}$, respectively. The anatomical group was significantly less than the non-anatomical group, with statistically significant difference $(\mathrm{P}<0.05)$.

\subsection{Liver Function Changes of HCC Patients in Both Groups on the 3rd Day after Surgery}

Table2. Changes in liver function of HCC patients in both groups on the 3rd day after surgery

\begin{tabular}{|c|c|c|}
\hline Observation target & AR group & NAR group \\
\hline ALT(U/L) & 213.35 & 415.46 \\
\hline AST(U/L) & 93.97 & 180.39 \\
\hline ALB $(\mathrm{g} / \mathrm{L})$ & 36.64 & 34.91 \\
\hline TBLL $(\mathrm{umol} / \mathrm{L})$ & 43.58 & 41.87 \\
\hline
\end{tabular}




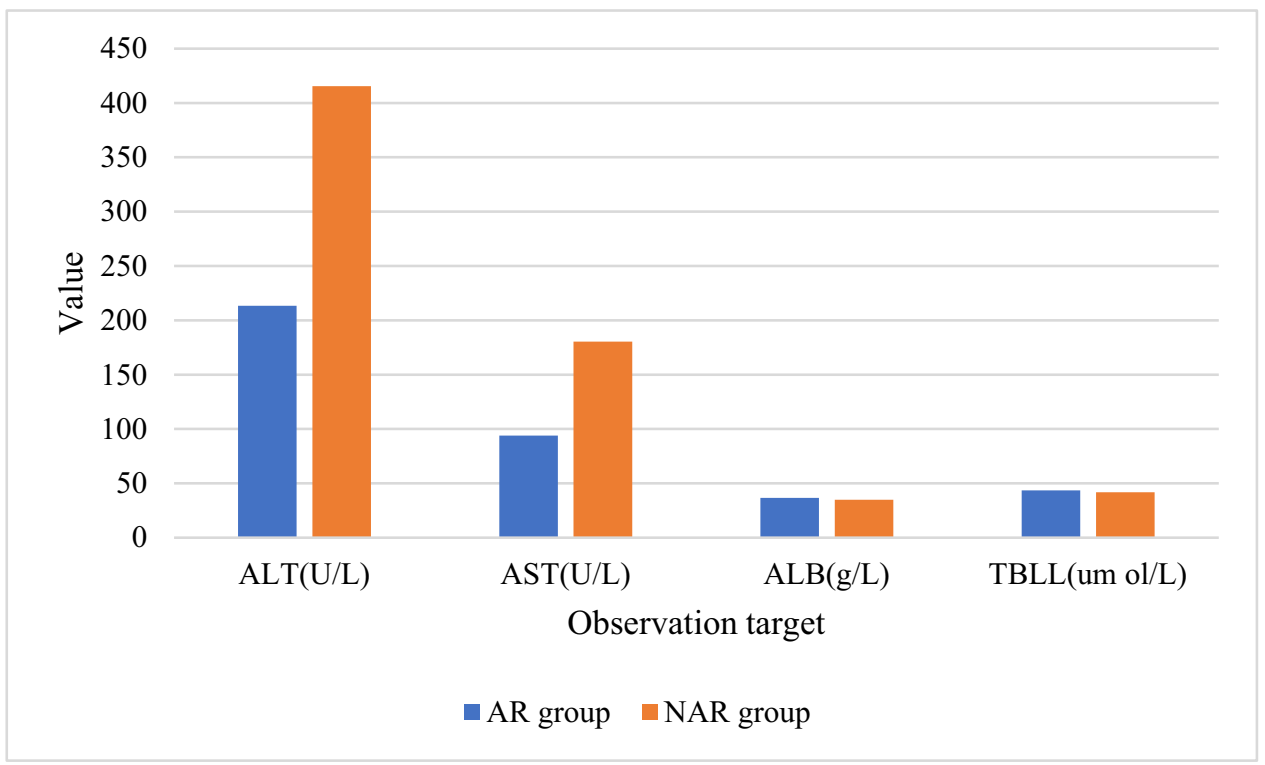

Figure1. Changes in liver function of HCC patients in both groups on the 3rd day after surgery

As shown in Table 2 and Figure 1, ALT, AST and ALB of the anatomic hepatectomy group were $213.35 \mathrm{U} / \mathrm{L}$, 93.97U/L and $36.64 \mathrm{~g} / \mathrm{L}$, respectively, 3 days after operation. ALT, AST and ALB were $415.46 \mathrm{U} / \mathrm{L}$, $180.39 \mathrm{U} / \mathrm{L}$ and $34.91 \mathrm{~g} / \mathrm{L}$, respectively, on the third day after operation in the non-anatomical hepatectomy group. After $\mathrm{T}$ test, the difference between the two groups was statistically significant $(\mathrm{P}<0.05)$. The total bilirubin
(TBIL) of the two groups was $43.58 \mathrm{umol} / \mathrm{L}$ and $41.87 \mathrm{umol} / \mathrm{L}$, respectively, on the third day after surgery. After $t$ test, the difference between the two groups was not statistically significant $(\mathrm{P}>0.05)$.

\subsection{Postoperative Complications of HCC Patients in the Two Groups}

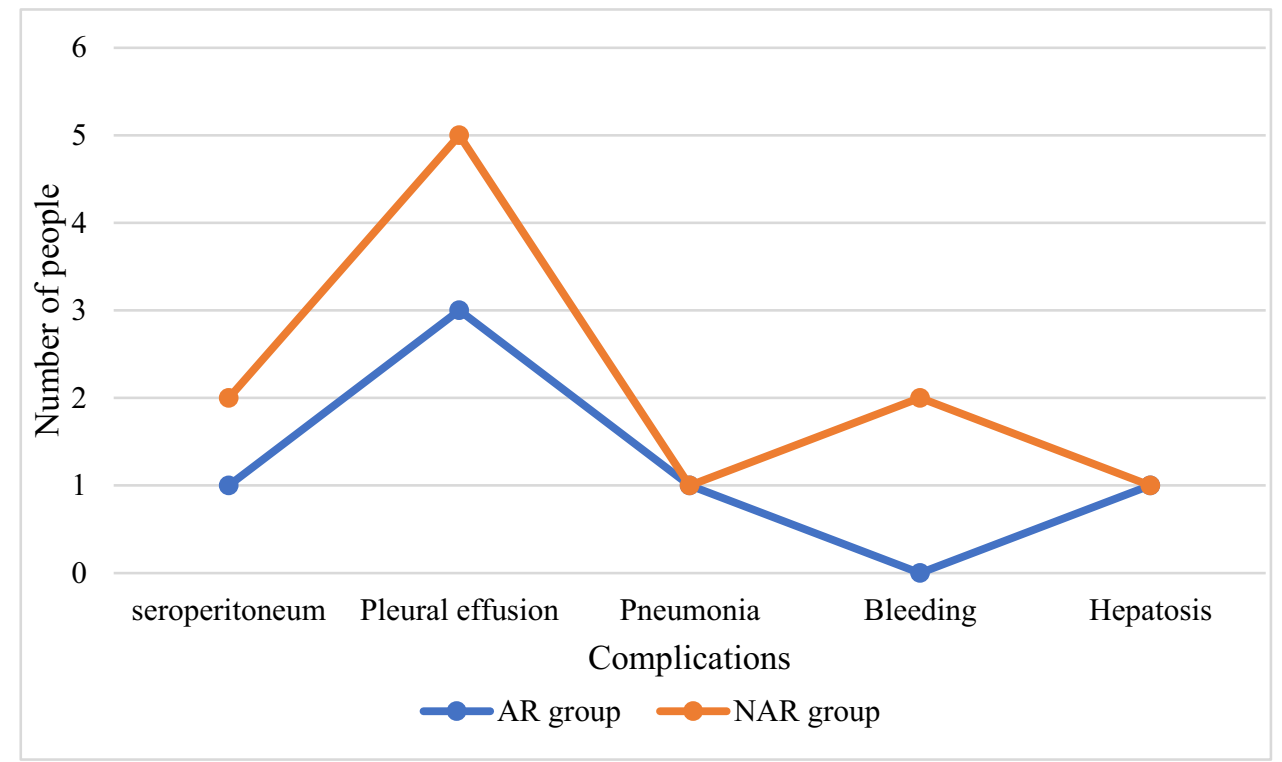

Figure2. Postoperative complications of HCC patients in the two groups

As shown in Figure 2, the incidence of postoperative complications in the anatomic hepatectomy group was $27.2 \%$, and that in the non-anatomic hepatectomy group was $55 \%$. After $\mathrm{X}$ test, the difference between the two groups was statistically significant $(\mathrm{P}<0.05)$. Both groups of patients with complications were cured and discharged after conservative treatment.

\section{Conclusions}

Compared with non-anatomical hepatectomy, anatomical hepatectomy has more complicated technical requirements and time-consuming operation, but its operation is delicate, less intraoperative bleeding and can reduce liver function damage, so it is relatively safe. Compared with non-anatomic hepatic resection, anatomic hepatic resection can reduce the early postoperative 
recurrence rate and improve the early postoperative disease-free survival rate. Early $\mathrm{HCC}$ recurrence is also closely related to AFP and microvascular invasion in peripheral blood. There are some deficiencies in this study: different clinicians conduct surgical management and postoperative management, resulting in inconsistent quality of patient care, which may affect the prognosis of patients. This study is a single-center retrospective study, with a small sample size and a certain degree of bias in data collection.

\section{Acknowledgment}

The successful completion of this thesis is due to my tutor's strict supervision, patient guidance and devoted help. I would like to thank my mentor for his advice and encouragement over the past seven years. Secondly, I would like to thank my Alma mater for giving me the opportunity to study here for seven years, so that I can continue to learn and make progress. Finally, I would like to thank my classmates for their care and help. I hope I can repay the cultivation of my Alma mater and mentor with practical actions in the future.

\section{References}

1. Cong $\mathrm{W} \mathrm{M}, \mathrm{Bu} \mathrm{H}$, Chen J, et al. Practice guidelines for the pathological diagnosis of primary liver cancer: 2015 update[J]. World Journal of Gastroenterology, 2016, 22(42):9279-9287.

2. Kon T, Suzuki H, Kawaguchi T, et al. Anatomical hepatectomy for liver metastasis from rectal adenocarcinoma presenting with intrabiliary extension: a case report[J]. Journal of Rural Medicine, 2016, 11(2):63-68.

3. Nanashima A, Nagayasu T. Development and clinical usefulness of the liver hanging maneuver in various anatomical hepatectomy procedures[J]. Surgery Today, 2016, 46(4):398-404.

4. Autran M, Machado C, Fábio F. Makdissi, et al. Laparoscopic right hepatectomy[J]. Revista Do Colégio Brasilro De Cirurgies, 2017, 34(3):189-192.

5. Qiu J, Chen S, Chengyou D. A systematic review of robotic-assisted liver resection and meta-analysis of robotic versus laparoscopic hepatectomy for hepatic neoplasms[J]. Surgical Endoscopy, 2016, 30(3):862875.

6. Song J, Wang Y, Ma K, et al. Laparoscopic hepatectomy versus radiofrequency ablation for minimally invasive treatment of single, small hepatocellular carcinomas.[J]. Surgical Endoscopy, 2015, 30(10):1-9.

7. Lai C, Jin R A, Liang $\mathrm{X}$, et al. Comparison of laparoscopic hepatectomy, percutaneous radiofrequency ablation and open hepatectomy in the treatment of small hepatocellular carcinoma[J]. Journal of Zhejiang Universityence B, 2016, 17(3):236-246.

8. Tanaka T, Iwasaki T, Takebe A, et al. Usefulness of Preoperative CT Simulation for Anatomical Hepatectomy of Liver Metastases in a Patient with Right Sided Ligamentum Teres[J]. Japanese Journal of Gastroenterological Surgery, 2017, 50(1):18-25.

9. Xu L N, Xu Y Y, Gao D W. Impact of operative and peri-operative factors on the long-term prognosis of primary liver cancer patients undergoing hepatectomy $[\mathrm{J}]$. Journal of Huazhong University of ence and Technology, 2016, 36(4):523-528.

10. Ryu T, Honda G, Kurata M, et al. Perioperative and oncological outcomes of laparoscopic anatomical hepatectomy for hepatocellular carcinoma introduced gradually in a single center[J]. Surgical Endoscopy, 2018, 32(2):790-798. 